\title{
Preterm Birth Reduces Nutrient Absorption With Limited Effect on Immune Gene Expression and Gut Colonization in Pigs
}

Østergaard, Mette V; Cilieborg, Malene S.; Skovgaard, Kerstin; Schmidt, Mette; Sangild, Per T.; Bering, Stine B.

Published in:

Journal of Pediatric Gastroenterology and Nutrition

Link to article, DOI:

$10.1097 / \mathrm{mpg} .0000000000000827$

Publication date:

2015

Document Version

Peer reviewed version

Link back to DTU Orbit

Citation (APA):

Østergaard, M. V., Cilieborg, M. S., Skovgaard, K., Schmidt, M., Sangild, P. T., \& Bering, S. B. (2015). Preterm Birth Reduces Nutrient Absorption With Limited Effect on Immune Gene Expression and Gut Colonization in Pigs. Journal of Pediatric Gastroenterology and Nutrition, 61(4), 481-490.

https://doi.org/10.1097/mpg.0000000000000827

\section{General rights}

Copyright and moral rights for the publications made accessible in the public portal are retained by the authors and/or other copyright owners and it is a condition of accessing publications that users recognise and abide by the legal requirements associated with these rights.

- Users may download and print one copy of any publication from the public portal for the purpose of private study or research.

- You may not further distribute the material or use it for any profit-making activity or commercial gain

- You may freely distribute the URL identifying the publication in the public portal 


\section{Preterm birth reduces nutrient absorption with limited effect on immune gene expression and gut colonization in pigs}

Mette Viberg Østergaard, $\mathrm{PhD}^{1 \#}$; Malene Skovsted Cilieborg ${ }^{1,2 \#}$, PhD; Kerstin Skovgaard, $\mathrm{PhD}^{2}$; Mette Schmidt, DVM, $\mathrm{PhD}^{1}$; Per Torp Sangild; PhD, DVSc, DMSc ${ }^{1}$; Stine Brandt Bering, $\mathrm{PhD}^{1}$.

${ }^{1}$ Departments of Nutrition, Exercise and Sports / Veterinary Clinical and Animal Sciences / Large Animal Sciences, University of Copenhagen, Frederiksberg C, Denmark. ${ }^{2}$ Innate Immunology Group, National Veterinary Institute, Technical University of Denmark, Frederiksberg C, Denmark. \# Both authors have contributed equally and may be considered as first authors.

The authors have no conflicts of interest.

\section{Financial statement:}

The study was supported by The Danish Research Councils.

\section{Corresponding author:}

Stine B. Bering, Department of Veterinary Clinical and Animal Sciences, Faculty of Health and Medical Sciences, University of Copenhagen, 68 Dyrlægevej, DK-1870 Frederiksberg C, Denmark; tel. +45 3533 10 92; fax +45 3533 24 83; E-mail: sbb@sund.ku.dk.

Running title: Prematurity, enteral diet and gut colonization

Key words: prematurity, diet, gut microbiota, intestinal function, innate immunity 
Author contribution: MVØ, MSC, PSA, and SBB designed the study; MS and PSA contributed with animal experimentation, MVØ, MSC, KS, and SBB contributed with data acquisition and analyses; MVØ, MSC, PSA, and SBB interpreted the data for the work; MVØ, MSC, and SBB drafted the manuscript; MVØ, MSC, KS, PSA, and SBB revised the manuscript critically for important intellectual content; MVØ, MSC, MS, KS, PSA, and SBB approved the final manuscript version to be published. 


\section{Abstract}

Introduction: The primary risk factors for necrotizing enterocolitis (NEC) are preterm birth, enteral feeding and gut colonization. It is unclear if feeding and colonization induce excessive expression of immune genes that lead to NEC. Using a pig model, we hypothesized that reduced gestational age would up-regulate immune-related genes and cause bacterial imbalance after birth.

Methods: Preterm (85-92\% gestation, $\mathrm{n}=53$ ) and near-term $(95-99 \%$ gestation, $\mathrm{n}=69)$ pigs were delivered by cesarean section and euthanized at birth or after two days of infant formula or bovine colostrum feeding.

Results: At birth, preterm delivery reduced 5 of 29 intestinal genes related to nutrient absorption and innate immunity, relative to near-term pigs, whereas two genes were up-regulated. Preterm birth also reduced ex vivo intestinal glucose and leucine uptake (40-50\%), but failed to increase cytokine secretions from intestinal explants relative to near-term birth. After two days of formula-feeding, NEC incidence was increased in preterm vs. near-term pigs (47 versus 0-13\%). Six of 29 genes related to immunity (TLR2, IL1B, IL8), permeability (CLDN3, OCLN) and absorption (SGLT) decreased in preterm pigs without affecting gram-negative bacteria related responses (TLR4, IKBA, NFkB1, TNFAIP3, PAFA). Bacterial abundance tended to be higher in preterm vs. near-term pigs $(P=0.09)$, whereas the composition was unaffected.

Conclusion: Preterm birth predisposes to NEC and reduces nutrient absorption, but does not induce upregulation of immune-related genes or cause bacterial dyscolonization in the neonatal period. Excessive inflammation and bacterial overgrowth may occur relatively late in NEC progression in preterm neonates. 


\section{Introduction}

Preterm infants are at risk of developing necrotizing enterocolitis (NEC), an acute gastrointestinal condition characterized by severe inflammation and necrosis. The risk of NEC is inversely correlated with gestational age and body weight at birth (1). Since preterm infants are born with an immature gastrointestinal tract with compromised motility, secretion, digestion, absorption and barrier functions, NEC is also associated with the introduction of enteral feeding, particularly when mother's milk is not available and formula feeding is needed (2). Gut barrier failure and abnormal bacterial colonization of the immature intestine may lead to excessive inflammatory responses that directly lead to NEC $(3,4)$. Thus, compromised intestinal homeostasis may explain why preterm infants are at particular risk of developing NEC, but the exact interactions between the compromised intestinal functions and mucosal immunity, diet and gut colonization after reduced gestational age remains unknown.

Previous studies have indicated that the immature NEC-sensitive intestine is characterized by an exaggerated inflammatory response to microbe-associated molecular patterns (MAMPs) and excessive IL-8 secretion from intestinal epithelial cells (IECs) $(5,6)$. The underlying mechanisms may involve enhanced expression and activation of toll-like receptor (TLR) $4(7,8)$ and reduced expression of negative regulators of TLR4 signaling pathways $(6,9)$, leading to prolonged activation of NFkB and production of pro-inflammatory mediators $(10,11)$. The results are based on studies in full-term mice and rats $(9,12)$, where NEC is typically induced by combining hypoxia and formula-feeding, or by studying human fetal intestines collected in weeks 12-20 of gestation, i.e. long before NEC development (6). A recent study have though shown decreased intestinal intensity and surface to cytosol ratio of TLR-4 from 16 to 21 weeks of human gestation (13). It remains to be established whether these exaggerated inflammatory responses are characteristic also for the viable ( $>24$ weeks) NEC-sensitive human fetus. Furthermore, as the majority of the findings described above have been generated in experimental NEC models, they may reflect molecular endpoints of NEC pathology more 
than predisposing factors that lead to NEC. Studies suggest that the unique role of TLR4 and an exaggerated expression of immune-related genes proposed from rodent studies could be less important in preterm NEC-susceptible pigs (14-17). However, it remains difficult to define the special characteristics of the immature NEC-sensitive intestine without comparing preterm and term individuals, reared in the same environment and fed the same diets.

The preterm pig born at $85-92 \%$ of gestation ( $\mathrm{d}$ 100-106, term $\mathrm{d}$ 116-117) is viable with moderate clinical support and may be comparable to a human preterm infant born at 70-80\% (28-32 weeks) of gestation with regard to gastrointestinal functions(18). Compared with pigs born close to term (e.g. 9599\% of gestation), preterm pigs are at high risk of developing NEC-like lesions following enteral formula feeding and bacterial colonization $(19,20)$, especially with a preceding period of total parenteral nutrition. Therefore, the preterm pig is a relevant animal model to study postnatal gut development and a clinically important animal model to investigate the responses of the immature intestine to bacterial colonization and enteral feeding, and their association with spontaneous NEC development (18). The preterm pig is the only existing model of preterm infants that can be easily used to study the influence of different degrees of prematurity and the relation to feeding regimens (e.g. time, dose and composition of parenteral and enteral feeding) and bacterial colonization (e.g. use of pre-, pro- and antibiotics).

Considering the complexity in understanding how prematurity, enteral feeding and gut colonization predispose to NEC, the aim of this study was to compare intestinal immune-related gene expressions in preterm pigs that have prematurity-related high NEC-sensitivity (85-92\% gestation) with responses in pigs born closer to term that are relatively NEC-resistant (95-99\% gestation). We studied caesarean-delivered pigs at birth (prior to enteral feeding and gut colonization) and after two days of enteral feeding and the associated colonization with bacteria from the environment. Because bovine colostrum has previously been documented to protect against NEC, relative to infant formula, we fed 
both these diets to help differentiate the immune gene responses related to diet and to gestational age at birth. We hypothesized that preterm birth would reduce the expression of genes involved in digestive (enzymes and transporters) and barrier functions (mucus, occludins and claudins) while innate immunity genes (pro- and anti-inflammatory cytokines, the TLR4 signaling complex and inhibitors of the NFKB signaling pathway) would be increased, consistent with the theory of exaggerated immune response to colonizing bacteria in preterm neonates. We also measured ex vivo intestinal cytokine secretion and nutrient uptake, and bacterial colonization of the small intestinal was analyzed by 454 pyro-sequencing of luminal contents and fluorescence in situ hybridization of tissue sections. With the used feeding protocol, without a preceding period of parenteral nutrition, we predicted a moderate NEC incidence (20-40\%) in formula-fed pigs (18). The experimental approach enabled us to study both the endogenous characteristics of the preterm pig intestine at birth and the intestinal responses to the first diet and gut colonization that may precede NEC.

\section{Methods}

\section{Animal procedures}

All animal procedures were approved by the Danish National Committee on Animal Experimentation.

Totally, 122 viable pigs (Danish Landrace X Large White X Duroc) were delivered by cesarean section from nine sows at d 100-106 of gestation (preterm, 85-92\% gestation; $\mathrm{n}=53$ ) or $\mathrm{d}$ 110-116 of gestation (near-term, 95-99\% gestation; $\mathrm{n}=69$ ) and transferred to heated and oxygenized incubators. To investigate the effect of gestational age on intestinal functions and gene expression independent of environmental factors, unfed preterm $(n=17)$ and near-term $(n=25)$ pigs were euthanized immediately after birth for tissue collection. Segments from the proximal, mid and distal small intestine were immediately prepared for ex vivo cytokine secretion and nutrient uptake studies or snap frozen in liquid nitrogen and stored at $-80^{\circ} \mathrm{C}$ for gene expression analyses. 
For $2 \mathrm{~d}$ old pigs, the rearing conditions, feeding regimen and NEC evaluation followed the methods previously described, without an initial period of parenteral nutrition (21). Briefly, pigs were kept in incubators, fitted with an orogastric feeding tube, and fed immediately after birth with formula (preterm formula, $\mathrm{n}=18$; near-term formula, $\mathrm{n}=23$ ) made from three commercially available products [Peptide 2-0 and Liquigen-MCT (SHS International, Liverpool, UK), Variolac and Lacprodan (Arla Foods Ingredients, Aarhus, Denmark) to a final concentration of $4081 \mathrm{~kJ} / \mathrm{L}$ containing 64 g protein/L, $47 \mathrm{~g}$ carbohydrate/L and $59 \mathrm{~g}$ fat/L] or bovine colostrum (preterm colostrum, $\mathrm{n}$ = 18; near-term colostrum, $n=21$, collected from a series of Holstein Friesian cows within $24 \mathrm{~h}$ of parturition, pooled and kept frozen until use) for $2 \mathrm{~d}$ before euthanasia and tissue collection. At euthanasia, five gastrointestinal regions (stomach, proximal, middle, and distal small intestine, and colon) were evaluated for macroscopic NEC-like lesions using a severity score from 1 to 6 , where 1 was assigned to healthy tissue and 6 to extensive hemorrhagic necrosis and pneumatosis intestinalis (22). NEC was defined as a score of minimum 3 in minimum one region. Contents and tissues from the distal small intestine were snap frozen and stored at $-80^{\circ} \mathrm{C}$ for later microbiology and gene expression analyses, respectively.

\section{Intestinal gene expression}

Expression of 29 genes related to innate immunity, digestive and barrier function was analyzed for the distal small intestine in both newborn unfed and $2 \mathrm{~d}$ old fed pigs. In order to obtain optimal RNA quality, only sections from fed pigs without obvious NEC lesions were included in the analysis. Frozen intestinal tissue samples were transferred to gentleMACS M tubes (MACS, Miltenyi Biotec, Lund, Sweden) containing QIAzol (Qiagen, Ballerup, Denmark) and homogenized using the gentleMACS Dissociator (MACS, Miltenyi Biotec). Total RNA was extracted with chloroform, precipitated with ethanol, and subsequently isolated using the RNeasy Midi kit (Qiagen) including on-column digestion 
of DNA using the RNase-Free DNAse set (Qiagen) according to manufacturer's protocol. RNA purity was assessed on a GeneQuant Pro spectrophotometer (Pharmacia Biotech, Cambridge, UK). cDNA synthesis was performed with 500 ng total RNA per sample using the QuantiTect Reverse Transcription Kit (Qiagen) according to manufacturer’s protocol. For assay validation, two cDNA synthesis reactions were prepared per samples. Non-reverse transcriptase controls were prepared for assessment of potential genomic DNA contamination.

Genes analyzed and primers applied are presented in Table 1. The genes are hereinafter referred to by their gene symbols. Primers were designed using Primer3 (http://frodo.wi.mit.edu/) (23) and synthesized at TAG Copenhagen (Copenhagen, Denmark). Amplification efficiencies, dynamic range, and specificity were estimated based on four separate dilution series of pooled cDNA and melting curve profiles. Gene expression was analyzed by quantitative real-time PCR (qPCR) performed in 48.48 Dynamic Array Integrated Fluidic Circuits (Fluidigm, South San Francisco, CA, USA) following the protocol and cycling parameters described previously (24).

Expression data (Cq values) were acquired using the Fluidigm Real-Time PCR Analysis software 3.0.2 (Fluidigm) and exported to GenEx (MultiD, Göteborg, Sweden) for data pre-processing including individual correction for PCR efficiency for each primer assay, normalizing to six highly stable reference genes (ACTB, GAPDH, HRPT1, RPL13A, TBP, and YWHAE), identified using GeNorm (25) and NormFinder (26), and averaging of cDNA technical repeats. Analysis of gene expression was run separately for newborn and $2 \mathrm{~d}$ old pigs, and the mean relative expression level of each primer assay was set to 1 in the preterm formula group, respectively, and all other groups were scaled accordingly.

Ex vivo nutrient uptake and cytokine secretion

D-Glucose and L-leucine uptake was measured ex vivo as previously described (14). Briefly, immediately after euthanasia, intact segments of the proximal small intestine were everted and mounted 
as 1-cm sleeves on steel rods with silk ligatures, while kept in ice-cold aerated Ringer's solution. The sleeves were pre-incubated for $12 \mathrm{~min}$ in $37^{\circ} \mathrm{C}$ aerated Ringer's solution, followed by incubation in an uptake solution for $2 \min \left[37^{\circ} \mathrm{C}\right.$ aerated Ringer's solution with $0.4 \mu \mathrm{M}$ [U- $\left.{ }^{14} \mathrm{C}\right]-\mathrm{D}-$ glucose and $5 \mathrm{nM}$ $\left[{ }^{3} \mathrm{H}\right]$-L-leucine; (Perkin Elmer, Skovlunde, Denmark)]. Finally, sleeves were washed in ice-cold Ringer’s solution, solubilized in Solvable (Perkin Elmer), and counted with Ultima Gold (Perkin Elmer) in a Tricarb 2100TR Liquid Scintillation Analyzer (Packard Instruments, Meriden, CT, USA).

To analyze ex vivo cytokine secretion, 10-15 cm segments were dissected from the middle jejunum from each pig, and submerged in ice-cold phosphate-buffered saline (PBS; 75\% v/v): Dulbecco’s modified Eagle medium (DMEM; 25\% v/v) supplemented with 1\% fetal bovine serum (FBS), $5 \mu \mathrm{g} / \mathrm{ml}$ gentamycin, and $1.25 \mu \mathrm{g} / \mathrm{ml}$ amphotericine B (Gibco, Life Technologies, Nærum, Denmark). The segments were flushed with $1 \mathrm{mM}$ dithiothreitol in PBS and subsequently PBS, and transferred to $37^{\circ} \mathrm{C}$ enriched DMEM [supplemented with 2\% FBS, $5 \mu \mathrm{g} / \mathrm{ml}$ gentamycine, $1.25 \mu \mathrm{g} / \mathrm{ml}$ amphotericine B, 100 U/ml penicillin (Gibco), 100 g/ml streptomycin (Gibco), 4.2 mM L-glutamine (Sigma-Aldrich, Brøndby, Denmark), and $1 \mathrm{mM}$ sodium pyruvate (Sigma-Aldrich)]. The segments were then opened longitudinally, cut into small explants of 2-4 mm $\times 2-4 \mathrm{~mm}$, and incubated for $2-4 \mathrm{~h}$ at $37^{\circ} \mathrm{C}$ and $5 \%$ $\mathrm{CO}_{2}$ in a humidified atmosphere. Explants were then transferred to 96-well plates with $200 \mu \mathrm{l} /$ well enriched DMEM (without FBS) with or without MAMPs [10 ng/ml lipopolysaccharide (LPS; from E. coli O26:B6, Sigma-Aldrich), 10 ng/ml flagellin (from S. typhimurium, Invivogen, Toulouse, France), or $1 \mu \mathrm{g} / \mathrm{ml} \mathrm{N}$-acetylmuramyl-L-alanyl-D-isoglutamine-hydrate (MDP; Sigma-Aldrich)]. Explants were incubated for $24 \mathrm{~h}$ at $37^{\circ} \mathrm{C}$ and $5 \% \mathrm{CO}_{2}$ in a humidified atmosphere after which culture supernatants were collected, centrifuged at $12,000 \times \mathrm{g}, 4^{\circ} \mathrm{C}$ for $10 \mathrm{~min}$, and stored at $-80^{\circ} \mathrm{C}$. The concentration of IL$1 \beta$, IL-6, IL-8 and TNF- $\alpha$ in the explants culture supernatants were quantified using Porcine IL-1 $\beta$, IL6, IL-8 and TNF-alpha DuoSet ELISA Development kits (R\&D Systems, Abingdon, United Kingdom) 
according to the manufacturer's protocol. Cytokine secretion was normalized to explant tissue weight thereby obtaining the total amount of cytokine secreted per tissue mass (pg/mg tissue).

\section{Microbiology}

The microbial composition of the distal small intestine was analyzed by 454 pyro sequencing. Samples were homogenized by bead beating with stainless steel beads for $2 \mathrm{~min} / 20 \mathrm{~Hz}$ in a tissue lyser (Qiagen). After DNA purification with the AllPrep DNA/RNA mini kit (Qiagen), the V4-V5 region of bacterial 16s rDNA was amplified using barcoded primers (5: 5’-CAGCAGCCGCGGTAATAC-3’', 926R 5’-CCGTCAATTCCTTTGAGTTT-3')(27). DNA quantity and quality was analyzed in a Agilent 2100 Bioanalyzer (Agilent Technologies, Waldbronn, Germany) using Agilent RNA 1000 Nano Kit, and 50 ng DNA from each of the 64 samples were pooled for sequencing on half a picoplate on 454 Roche GS FLX titanium platform at LGC Genomics (Berlin, Germany) following the standard amplicon sequencing protocols (Roche, Basel, Switzerland). The dataset was analyzed in the Quantitative Insight Into Microbial Ecology (QIIME) open source software package (28). Raw data underwent quality control, de-noising, chimera filtering and Operational Taxonomic Unit (OTU) picking as previously described (29) and the remaining sequences were clustered at $97 \%$ relatedness using UCLAST (http://www. drive5.com/usearch/). Representative sequences from each cluster were aligned with pyNAST (http://qiime.org/pynast/), subjected to the Ribosomal Database Project (RDP)based 16S rRNA gene annotation. Alpha diversity was calculated as Observed species; Chao1 and Shannon indexes for OTU tables that were unified to 1000 sequences per sample. Principal Coordinate Analysis (PCoA) plots were generated with the Jackknifed Beta Diversity workflow based on 10 distance metrics calculated using 10 subsampled OTU tables and 85\% of sequences taken for each jackknifed subset. 
Bacterial abundance was evaluated by fluorescence in situ hybridization (FISH) on paraffin embedded cross sections (3 $\mu \mathrm{m})$ of nine sections along the distal small intestine with Alexa fluor 555labeled oligonucleotide probes (Eurofins MWG Operon, Ebersberg, Germany) targeting 16S rRNA of general bacteria (5'-GCTGCCTCCCGTAGGAGT-3’)(30) and visualized using an Axioimager M1 epifluorescence microscope (Carl Zeiss, Oberkochen, Germany) equipped for epifluorescence and filter set XF53 (Omega Optical, Brattleboro, Vt., USA). Based on the red fluorescence signal, the tissue sections were scored from 0 to 6, where 0: 0-9 bacteria in a tissue section, 1: 10-49 spread bacteria, 2: small colonies (50-299), 3: few larger colonies (300-500), 4: several larger colonies, 5: numerous larger colonies, and 6: widespread overgrowth with large colonies.

\section{Statistical analyses}

Nutrient uptake data were analyzed using a t-test in GraphPad Prism (version 6.01, GraphPad Software, La Jolla, CA, USA). Remaining analyses were performed in R (version 2.15.0). Data on gene expression and intestinal explants were modeled in mixed linear models using the lmer function (package Ime4). Gene expression data were $\log _{2}$-transformed before modeling and the model included "gestational age” and “diet” as fixed variables and "litter" as a random variable. A fold-change of $\geq 1.5$ (linear scale) were defined as a cutoff for biologically significance. Data from intestinal explants were transformed before analyses using the survreg function (package survival) and the model included “gestational age” and “MAMP treatment” as fixed variables and "litter” and “pig” as random variables. For 454 sequencing data, group differences in the PCoA plots were evaluated by Analysis of similarities (ANOSIM) using weighted and unweighted uniFrac distance metrics based on rarefied OTU tables (excluding 7 samples with less than 1000 reads per sample). Differences in the 15 most dominating OTUs were tested using the Imer function (lme4 package) in R (version 3.0.1, open source software, available online: cran.r-project.org), where “litter” was a random variable and “treatment”, 
“diet” and "gestational age” were fixed variables. $P=0.05$ was used as the significance level. NEC incidences and FISH scores were analyzed with logistic regression ( $\mathrm{glm}$ function) and repeated measurements (lme function), respectively, using “gestational age” and “diet” as fixed variables and “litter” as random variable. All data presented in tables and figures are mean \pm SEM. The resulting $P$ values are evaluated at a 5\% significance level.

\section{Results}

Intestinal gene expression in newborn unfed pigs

Seven of the tested genes were differentially expressed between newborn unfed preterm and near-term pigs (Fig. 1). The expression of genes related to nutrient digestion and absorption were analyzed as markers of intestinal digestive function. The intestinal expression of LCT (lactase-phloridzin hydrolase, lactose-digesting enzyme) and SGLT1 (sodium glucose linked transporter 1, glucose transporter) was 47\% and 84\% higher, respectively, in near-term vs. preterm pigs ( $P<0.01$ and $P<0.001$, respectively). The expression of PRDM1, a marker of enterocyte immaturity, was not different between groups. To evaluate intestinal mucosal immunity, the expression of genes related to epithelial barrier function, cytokines and LPS-signaling was analyzed in the small intestine. The expression of MUC1 (coding for a mucus protein) was reduced with $40 \%$ in near-term vs. preterm pigs ( $P<0.05)$, while $M U C 2$ and the tight junction proteins CLDN3 and OCLN did not differ between gestational ages. Intestinal expression of $I L 6$ was reduced with $41 \%(P<0.001)$ and $I L 8$ increased with $69 \%(P<0.05)$ in near-term relative to preterm pigs, while the expression of $I L 1 B$, TNFA or TGFB did not differ between gestational ages. We did not observe differences in the expression of genes related to the TLR4-signaling complex including TLR4, MD2 and CD14 nor in the expression of inhibitors of the NFאB signaling pathway (IKBA, TNFAIP3 and PPARG) between preterm and near-term pigs. Intestinal gene expression $L B P$, coding for an acute-phase protein, and IAP, coding for the multifunctional intestinal alkaline 
phosphatase protein involved in mucosal protection, were 5.5- and 3-fold higher, respectively, in the near-term vs. preterm pigs $(P<0.001$ and $P<0.01$, respectively).

Intestinal ex vivo nutrient uptake and cytokine secretion in newborn unfed pigs

Intestinal nutrient absorption capacity was assessed ex vivo as a functional marker of intestinal maturity. The relative absorption of D-glucose and L-leucine was reduced by $\sim 38 \%$ and $\sim 47 \%$, respectively, in the proximal small intestine in preterm vs. near-term pigs $(P<0.05$ and $P<0.01$, respectively, Fig. 2A).

Secretion of pro-inflammatory cytokines was quantified ex vivo in small intestinal explants from preterm and near-term pigs with and without MAMP induction (Fig. 2B-C). Basal secretion of IL-6, IL-8 and TNF- $\alpha$ did not differ between preterm and near-term pigs (Fig. 2B), nor were there any changes after MAMP stimulation in IL-8 (Fig. 2C), IL-6 and TNF- $\alpha$ secretion (data not shown).

\section{Clinical status of two day old fed pigs}

Mean birth weight for both unfed and fed pigs was $0.92 \mathrm{~kg}$ for preterm and $1.35 \mathrm{~kg}$ for near-term pigs $(P<0.05)$ with no difference between the diet groups. During the $2 \mathrm{~d}$ study period, the preterm pigs lost more weight than the term pigs (-148 and $-120 \mathrm{~g} / \mathrm{kg}$ for preterm formula and colostrum pigs, respectively vs. -92 and $-71 \mathrm{~g} / \mathrm{kg}$ for corresponding near-term pigs, $P<0.05$ ).

Following euthanasia and tissue evaluation, eight pigs were diagnosed with mild NEC lesions, and NEC was more prevalent in preterm formula pigs (47\%) compared with the other three groups (preterm colostrum, near-term formula and near-term colostrum pigs, 0-15\% NEC, $P<0.05)$. Consequently, the average lesion score across five gastrointestinal regions was higher for preterm formula pigs than for the other groups $(2.1 \pm 0.2$ vs. $1.3 \pm 0.2,1.3 \pm 0.2$ and $1.2 \pm 0.2$ for the preterm colostrum, near-term formula and near-term colostrum pigs, respectively, $P<0.05)$. 
Intestinal gene expression in two day old fed pigs

After $2 \mathrm{~d}$ of formula or colostrum feeding, 10 of the investigated intestinal genes related to nutrient digestion and uptake, mucosal barrier function and immunity were independently affected, either by gestational age or diet (Fig. 3). The intestinal expression of genes related to immunity pathways TLR2, $I L 1 B, I L 8$, permeability CLDN3, OCLN and nutrient absorption SGLT were decreased by $45-65 \%$ in preterm vs. near-term pigs. Compared with colostrum, formula feeding increased the expression of MUC2 by 50\% $(P<0.05)$, whereas it decreased the intestinal expression of the complement factor $C 3$ ( $P<0.001)$, IAP $(P<0.01)$ and $L C T(P<0.05)$ by $40-50 \%$. DEF2 and CCL5, involved in barrier function and T-cell activation, respectively, were not affected by gestational age or diet, and as for unfed pigs, there was no difference in the expression of genes related to the TLR4-signaling complex and NFkB signaling pathway, including TLR4, IKBA, NFkB1, TNFAIP3 and PAFA.

\section{Intestinal microbiota in fed pigs}

After quality control, de-noising and chimera removing, 454 pyro sequencing of 64 samples resulted in a total of 374140 sequences (range 40-16778) with a mean of $5846 \pm 533$ sequences per sample and no difference between groups. Totally, 52 different OTUs were identified with an average of $13.4 \pm 0.8$ different OUTs per sample. The 15 most dominating OTUs (Fig. 4A-C) accounted for $99.44 \%$ of the total sequences, where Clostridium was the most abundant genus with mean relative abundance of 34.0 $\pm 2.8 \%$ across samples and Enterococcus, the second most dominating genus, had a mean relative abundance of $22.2 \pm 3.5 \%$. There was considerable inter-individual variation in relative abundance of OTUs, however, mean relative abundance of Enterococcus was higher and Streptococcus lower in formula compared with colostrum pigs $(P<0.01$, Fig. 4A), while gestational age did not affect relative abundance of individual OTUs. Mean alpha diversity, indicating bacterial diversity in the four 
treatment groups, was increased after colostrum feeding compared to formula feeding (Fig 4B, $P<$ 0.01). Gestational age did not affect diversity $(P=0.2)$.

PCoA plots, both weighted (accounting for relative abundance) and unweighted (only accounting for presence or not), also demonstrated a diet-effect with separation between formula and colostrum pigs (both $P<0.01$, $\mathrm{R}=0.13$, Fig. 4C). There was no separation according to gestational age $(P=0.2$ and 0.8 for weighted and unweighted analyses, respectively, Fig. 4D) or individual groups (data not shown).

FISH analyses revealed relatively low bacterial abundance in the nine investigated regions of the small intestine (Fig. 5A-E), with total absence of bacteria in 30 and $60 \%$ of the sections from preterm and term pigs, respectively $(P<0.001)$, and a trend towards higher bacterial abundance across the entire small intestine in preterm pigs, compared with term pigs (Fig. 5A, $P=0.09$ ). Diet did not affect bacterial abundance.

\section{Discussion}

Preterm birth disrupts the normal prenatal maturation of many gut functions, specifically those that mature in the late gestation. This may explain why preterm infants show impaired nutrient absorption and develop adverse inflammatory responses to enteral feeding and bacterial colonization , potentially leading to severe feeding intolerance and NEC (2). However, it remains difficult to know the exact sequence of events, and to separate the early predisposing factors for NEC from the later molecular events associated with disease progression. This is important as steps to prevent NEC should attempt to prevent the early pathological events. Thus, intense research efforts have sought to clarify the intestinal molecular mechanisms and pathways that predispose to NEC. A dominating theory is that bacterial colonization of the preterm gut leads to an inappropriate excessive activation of the TLR4-complex and NFkB pathway $(5-7,31)$. Evidence for this theory originates mainly from rodent models of NEC and 
observations from infant fetal tissue $(6,12)$. It remains unclear whether these findings reflect the high NEC sensitivity in preterm infants born at different gestational ages, and whether the proposed mechanisms are central triggers of NEC or reflect events associated with NEC progression. Using pigs as models, we hypothesized that the well-known down-regulation of digestive function following preterm birth would be paralleled with an early up-regulation of genes related to innate immunity and inflammation, relative to pigs delivered close to term. Contrary to our hypothesis, we observed that there was no up-regulation of genes related to intestinal immunity, neither before nor just after the first enteral feeding and bacterial colonization. While a NEC-protective diet, like bovine colostrum, induced modest changes to the gut microbiota and reduced NEC, it did not reduce the expression of key genes related to immunity and inflammation. Thus, an excessive expression of intestinal immunity genes is not a key predisposing factor for NEC, at least not in pigs.

To study both the events that precede and lead to NEC, we used newborn, unfed pigs as well as pigs fed either colostrum or formula for two days. Since $44 \%$ of the preterm formula pigs and only 0 $13 \%$ of remaining pigs (preterm colostrum pigs, near-term pigs fed either diet) developed NEC, the present study confirms that our model is valid to study spontaneous NEC development in preterm neonates. It is important to note that the NEC lesions observed at autopsy mainly in $2 \mathrm{~d}$ old formula pigs (average NEC score 2.1) were relatively mild, representing the early stage of the disease, relative to the frequent observation of more severe NEC symptoms observed when using our standard $5 \mathrm{~d}$ protocol that includes a period of parenteral nutrition before introduction of formula $(15,22,32-34)$.

In newborn, unfed pigs seven of 29 investigated genes were affected by gestational age with higher expression of $L C T$, SGLT1, IL8, IAP and $L B P$, and lover expression of MUC2 and IL6, in near-term vs. preterm pigs, while expression of genes related to barrier function (MUC2, CLDN3, OCLN), and endotoxin-mediated TLR4-signaling (TLR4, MD2, CD14, IKBA, TNFAIP3, PPARG), were largely unaffected. Near-term pigs showed greater ex vivo absorption of glucose and leucine, while ex vivo 
secretion of the pro-inflammatory cytokines IL-6, IL-8 and TNF- $\alpha$ was unaffected by gestational age. After two days of feeding, expression of six genes (TLR2, IL1B, IL8, CLDN3, OCLN, SGLT1) was decreased in preterm vs. near-term pigs. The type of diet affected four genes, which were either up(C3, MUC2) or down-regulated (IAP, LCT) by infant formula, compared with colostrum. Diet, but not gestational age at birth, influenced the microbial composition of the small intestine in $2 \mathrm{~d}$ old pigs, while prematurity, especially coupled with formula feeding, tended to increase the overall bacterial abundance. On the other hand, formula feeding significantly decreased bacterial diversity in both preterm and term pigs. Thus, we found that totally 11 of 29 investigated genes were affected by gestational age in newborn unfed and $2 \mathrm{~d}$ old fed pigs, and that four genes were affected by diet in $2 \mathrm{~d}$ old pigs. However differences were modest ( $0.5-2.5$ fold), except for $L B P$ and IAP in unfed pigs, and our results therefore do not support our hypotheses that preterm birth in itself leads to excessive expression of central genes related to mucosal immunity. As predicted, spontaneous NEC development occurred frequently in preterm formula-fed pigs, but rarely in near-term pigs or preterm pigs fed colostrum. While the genes and functions investigated in this study only represent a small part of those that have been suggested to be involved in NEC, and taking into account that there may not be direct correlation between the gene expression and the active protein levels, they do cover key elements, such as nutrient absorptive and barrier functions, as well as some of the well-known TLRs and inflammatory mediators.

We have previously documented that preterm birth is associated with reduced lactase activity $(14,35,36)$ and absorption of glucose and galactose (14), consistent with the reduced ex vivo nutrient uptake and expression of $L C T$ and SGLT1 in preterm vs. near-term pigs in this study. Thus, preterm infants suffer from nutrient malabsorption, leading to an accumulation of unabsorbed nutrients in the lower bowel, potentially having both direct epithelial effects and effects via increased bacterial fermentation. Both immune stimulation by bacteria and accumulation of their fermentation products 
may cause damage to the intestinal epithelium and predispose to NEC $(37,38)$. Accordingly, we have consistently observed in other studies that increased NEC incidence is associated with increased concentration of short chain fatty acids in the colon, especially of lactic acid $(17,32,34)$. The apparent consequences of low LCT expression in the immature intestine, together with impaired barrier and glucose absorptive function, and excessive nutrient fermentation, can be prevented by feeding a NECprotective diet, like bovine colostrum. This may be key to explain that mother's milk protect against NEC in preterm infants.

The expression of genes related to the LPS-receptor complex and of intracellular inhibitors of NFkB signaling did not differ between newborn pigs and $2 \mathrm{~d}$ old pigs fed colostrum or formula. This is consistent with previous studies in preterm pigs, showing that TLR4 expression was not increased at birth in healthy preterm pigs compared with term pigs (14), and that there was no association between NEC and enhanced expression of the TLR4-complex in the intestinal epithelium $(15,17)$. Further, our reported effects on gene expression related to inflammatory markers and the TLR4-complex have been rather modest with only 1.5-3 fold changes (15-17), in contrast to the marked changes observed in rodent models where NEC is induced postnatal in full-term animals $(8,39)$. It is noteworthy that infant NEC tissues did not show significantly up-regulated TLR-4 gene expression, compared with tissues from spontaneous intestinal perforation, and only modest up-regulation compared with control tissues (40). Also, TLR-4 appeared to exert a protective effect for ischemia-reperfusion intestinal injury in neonatal mice (41). Accordingly, we suggest that induced expression of the TLR4-complex and NFkB signaling, and resulting excessive intestinal inflammation, are not key elements in the progression of NEC in preterm pigs.

We observed that $L B P$ and IAP expression was greater in the intestine of near-term vs. preterm unfed pigs and that IAP expression was further increased after colostrum feeding in both preterm and near-term pigs. In neonatal rats, oral administration of LBP did not protect against experimentally 
induced NEC, but may attenuate LPS-mediated inhibition of IEC migration and wound healing in vitro (42). Furthermore, it has been shown, that iAP catalyzes detoxification of LPS in the small intestinal lumen and thereby maintains gut homeostasis (43), and that enteral administration of exogenous iAP protects against NEC neonatal rats (44), possibly through decreased expression of pro-inflammatory mediators and decreased intestinal permeability, as shown in immature newborn rats (45).

The fetal human intestine and immature IECs are characterized by exaggerated pro-inflammatory responses and excessive IL-8 secretion in response to MAMPs, IL-1 $\beta$ and TNF- $\alpha$. This may in part be

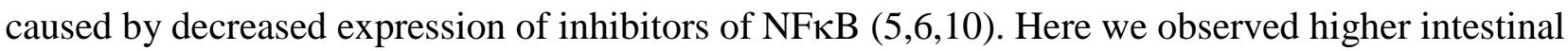
expression of $I L 8$ in both fed and unfed near-term vs. preterm pigs. This is consistent with the findings of increased expression of genes related to the IL-8 signaling pathways with advancing maturation of the fetal human intestine $(46,47)$. Following colonization and feeding, a well-controlled IL-8 release may interact with endotoxins and TGF $\beta$ to support epithelial repair, as suggested from recent studies in preterm pigs and epithelial cell lines (48). In contrast, IL6 expression was higher in unfed preterm vs. near-term pigs, suggesting different roles of these classical pro-inflammatory cytokines in prenatal intestinal development, and a more sensitive overexpression of IL6 in the premature state. Regardless, our studies on IL-6, IL-8 and TNF- $\alpha$ protein secretion from intestinal explant cultures indicate that the inflammatory response to the very first exposure to bacterial components is not markedly affected by gestational age at birth (preterm vs. near-term). Excessive, aberrant release of these cytokines are therefore not likely to be predisposing factors for NEC in pigs, supporting our previous finding that the TLR-4 signaling complex may not be a key pathway in NEC pathogenesis.

Gestational age at birth did not influence the microbiota composition and bacterial diversity as shown by 454 pyro sequencing. Rather, diet had a modest influence on both composition and diversity, with increased abundance of Enterococcus, decreased abundance of Streptococcus and decreased bacterial diversity after $2 \mathrm{~d}$ formula feeding. The dietary effect on gut colonization is in line with the 
general accepted hypothesis that feeding mother's milk, compared with formula feeding, improves infant gut colonization with more Bifidobacteria and Lactobacilli, and less potential pathogens (49). The result is, however, in contrast with a previous study using a similar experimental protocol and where prematurity and NEC, rather than environment and diet, influenced the microbial composition (21). These discrepancies may reflect a more detailed microbiology analysis with 454 pyro sequencing, compared with the T-RFLP analyses used previously. Bacterial abundance in situ did however support our previous study with a tendency to increased bacterial abundance in the small intestine of preterm versus near-term pigs, irrespective of diet (50). This correlates well with the finding that human tissues resected from NEC patients also show increased bacterial abundance, compared with non-NEC samples (51). This increased abundance was not, at least not initially, associated with increased expression of innate immunity genes. Possibly the compromised digestive and absorptive functions play a role to increase bacterial density along the mucosal surface and increase nutrient fermentation in NEC pigs, as shown in several of our earlier studies $(17,32,34)$.

The present study confirms that preterm formula-fed pigs are NEC-sensitive and show immature nutrient absorption, but this is not explained by a prematurity-stimulated excessive expression of key immune-related genes in the intestine. Initial gut colonization is influenced by diet but not by gestational age at birth, thus immaturity is not in itself enough to facilitate a NEC-related gut dyscolonization. Our study does not support the theory that factors involved in the TLR4-complex, NFkB signaling and excessive expression of inflammatory markers are key underlying factors in NEC progression in preterm neonates. The damaging effect of excessive bacteria-dependent nutrient fermentation, secondary to impaired digestive functions, may be more important. 


\section{Acknowledgements}

We thank Mandy Grieg from Department of Large Animal Sciences, and Elin Skytte from Department of Nutrition, Exercise and Sports, University of Copenhagen, Karin Tarp, Annie Ravn Pedersen and Sophia Rasmussen from National Veterinary Institute, Technical University of Denmark for technical support with animal procedures and laboratory analyses. We thank Łukasz Krych from Department of Food Science, University of Copenhagen, for performing microbiota analysis. We also thank Christian Ritz from Department of Nutrition, Exercise and Sports, University of Copenhagen, for statistical counseling. 


\section{References}

1. Fitzgibbons SC, Ching Y, Yu D et al. Mortality of necrotizing enterocolitis expressed by birth weight categories. J Pediatr Surg 2009;44(6):1072-1075.

2. Lin PW, Nasr TR, Stoll BJ. Necrotizing enterocolitis: recent scientific advances in pathophysiology and prevention. Semin Perinatol 2008;32(2):70-82.

3. Renz H, Brandtzaeg P, Hornef M. The impact of perinatal immune development on mucosal homeostasis and chronic inflammation. Nat Rev Immunol 2012;12(1):9-23.

4. Morowitz MJ, Poroyko V, Caplan M, Alverdy J, Liu DC. Redefining the role of intestinal microbes in the pathogenesis of necrotizing enterocolitis. Pediatrics 2010;125(4):777-785.

5. Claud EC, Savidge T, Walker WA. Modulation of human intestinal epithelial cell IL-8 secretion by human milk factors. Pediatr Res 2003;53(3):419-425.

6. Nanthakumar N, Meng D, Goldstein AM et al. The mechanism of excessive intestinal inflammation in necrotizing enterocolitis: an immature innate immune response. PLoS One 2011;6(3):e17776.

7. Leaphart CL, Cavallo J, Gribar SC et al. A critical role for TLR4 in the pathogenesis of necrotizing enterocolitis by modulating intestinal injury and repair. J Immunol 2007;179(7):48084820. 
8. Sodhi CP, Shi XH, Richardson WM et al. Toll-like receptor-4 inhibits enterocyte proliferation via impaired beta-catenin signaling in necrotizing enterocolitis. Gastroenterology 2010;138(1):185196.

9. Claud EC, Lu L, Anton PM, Savidge T, Walker WA, Cherayil BJ. Developmentally regulated IkappaB expression in intestinal epithelium and susceptibility to flagellin-induced inflammation. Proc Natl Acad Sci U S A 2004;101(19):7404-7408.

10. Claud EC, Zhang X, Petrof EO, Sun J. Developmentally regulated tumor necrosis factor-alpha induced nuclear factor-kappaB activation in intestinal epithelium. Am J Physiol Gastrointest Liver Physiol 2007;292(5):G1411-G1419.

11. De Plaen IG, Liu SX, Tian R et al. Inhibition of nuclear factor-kappaB ameliorates bowel injury and prolongs survival in a neonatal rat model of necrotizing enterocolitis. Pediatr Res 2007;61(6):716-721.

12. Gribar SC, Sodhi CP, Richardson WM et al. Reciprocal expression and signaling of TLR4 and TLR9 in the pathogenesis and treatment of necrotizing enterocolitis. J Immunol 2009;182(1):636646.

13. Meng D, Zhu W, Shi HN et al. Toll-like receptor-4 in human and mouse colonic epithelium is developmentally regulated: a possible role in necrotizing enterocolitis. Pediatr Res 2014.

14. Bering SB, Bai S, Zhang K, Sangild PT. Prematurity does not markedly affect intestinal sensitivity to endotoxins and feeding in pigs. Br J Nutr 2012;108(4):672-681. 
15. Siggers J, Sangild PT, Jensen TK et al. Transition from parenteral to enteral nutrition induces immediate diet-dependent gut histological and immunological responses in preterm neonates. Am J Physiol Gastrointest Liver Physiol 2011;301(3):G435-G445.

16. Cilieborg MS, Schmidt M, Skovgaard K et al. Fetal lipopolysaccharide exposure modulates dietdependent gut maturation and sensitivity to necrotising enterocolitis in pre-term pigs. Br J Nutr 2011;106(6):852-861.

17. Stoy AC, Heegaard PM, Thymann T et al. Bovine colostrum improves intestinal function following formula-induced gut inflammation in preterm pigs. Clin Nutr 2014;33(2):322-329.

18. Sangild PT, Thymann T, Schmidt M, Stoll B, Burrin DG, Buddington RK. Invited Review: The preterm pig as a model in pediatric gastroenterology. J Anim Sci 2013;91(10):4713-4729.

19. Jiang P, Wan JM, Cilieborg MS, Sit WH, Sangild PT. Premature delivery reduces intestinal cytoskeleton, metabolism, and stress response proteins in newborn formula-fed pigs. J Pediatr Gastroenterol Nutr 2013;56(6):615-622.

20. Sangild PT, Petersen YM, Schmidt M et al. Preterm birth affects the intestinal response to parenteral and enteral nutrition in newborn pigs. J Nutr 2002;132(12):3786-3794.

21. Cilieborg MS, Boye M, Molbak L, Thymann T, Sangild PT. Preterm birth and necrotizing enterocolitis alter gut colonization in pigs. Pediatr Res 2011;69(1):10-16. 
22. Jensen ML, Thymann T, Cilieborg MS et al. Antibiotics modulate intestinal immunity and prevent necrotizing enterocolitis in preterm neonatal piglets. Am J Physiol Gastrointest Liver Physiol 2014;306(1):G59-G71.

23. Skovgaard K, Mortensen S, Boye M et al. Rapid and widely disseminated acute phase protein response after experimental bacterial infection of pigs. Vet Res 2009;40(3):23.

24. Skovgaard K, Cirera S, Vasby D et al. Expression of innate immune genes, proteins and microRNAs in lung tissue of pigs infected experimentally with influenza virus (H1N2). Innate Immun 2013;19(5):531-544.

25. Vandesompele J, De PK, Pattyn F et al. Accurate normalization of real-time quantitative RT-PCR data by geometric averaging of multiple internal control genes. Genome Biol 2002;3(7):RESEARCH0034.

26. Andersen CL, Jensen JL, Orntoft TF. Normalization of real-time quantitative reverse transcription-PCR data: a model-based variance estimation approach to identify genes suited for normalization, applied to bladder and colon cancer data sets. Cancer Res 2004;64(15):5245-5250.

27. Nordentoft S, Molbak L, Bjerrum L, De VJ, Van IF, Pedersen K. The influence of the cage system and colonisation of Salmonella Enteritidis on the microbial gut flora of laying hens studied by T-RFLP and 454 pyrosequencing. BMC Microbiol 2011;11:187.

28. Caporaso JG, Kuczynski J, Stombaugh J et al. QIIME allows analysis of high-throughput community sequencing data. Nat Methods 2010;7(5):335-336. 
29. Bangsgaard Bendtsen KM, Krych L, Sorensen DB et al. Gut microbiota composition is correlated to grid floor induced stress and behavior in the BALB/c mouse. PLoS ONE 2012;7(10):e46231.

30. Molbak L, Johnsen K, Boye M et al. The microbiota of pigs influenced by diet texture and severity of Lawsonia intracellularis infection. Vet Microbiol 2008;128(1-2):96-107.

31. Hunter CJ, De Plaen IG. Inflammatory signaling in NEC: Role of NF-kappaB, cytokines and other inflammatory mediators. Pathophysiology 2014;21(1):55-65.

32. Jensen ML, Sangild PT, Lykke M et al. Similar efficacy of human banked milk and bovine colostrum to decrease incidence of necrotizing enterocolitis in preterm piglets. Am J Physiol Regul Integr Comp Physiol 2013;305(1):R4-R12.

33. Moller HK, Thymann T, Fink LN, Frokiaer H, Kvistgaard AS, Sangild PT. Bovine colostrum is superior to enriched formulas in stimulating intestinal function and necrotising enterocolitis resistance in preterm pigs. Br J Nutr 2011;105(1):44-53.

34. Bjornvad CR, Thymann T, Deutz NE et al. Enteral feeding induces diet-dependent mucosal dysfunction, bacterial proliferation, and necrotizing enterocolitis in preterm pigs on parenteral nutrition. Am J Physiol Gastrointest Liver Physiol 2008;295(5):G1092-G1103.

35. Sangild PT, Sjostrom H, Noren O, Fowden AL, Silver M. The prenatal development and glucocorticoid control of brush-border hydrolases in the pig small intestine. Pediatr Res 1995;37(2):207-212. 
36. Sangild PT, Schmidt M, Elnif J, Bjornvad CR, Westrom BR, Buddington RK. Prenatal development of gastrointestinal function in the pig and the effects of fetal esophageal obstruction. Pediatr Res 2002;52(3):416-424.

37. Buddington RK, Bering SB, Thymann T, Sangild PT. Aldohexose malabsorption in preterm pigs is directly related to the severity of necrotizing enterocolitis. Pediatr Res 2008;63(4):382-387.

38. Lin J. Too much short chain fatty acids cause neonatal necrotizing enterocolitis. Med Hypotheses 2004;62(2):291-293.

39. Liu Y, Zhu L, Fatheree NY et al. Changes in intestinal Toll-like receptors and cytokines precede histological injury in a rat model of necrotizing enterocolitis. Am J Physiol Gastrointest Liver Physiol 2009;297(3):G442-G450.

40. Chan KY, Leung KT, Tam YH et al. Genome-wide expression profiles of necrotizing enterocolitis versus spontaneous intestinal perforation in human intestinal tissues: dysregulation of functional pathways. Ann Surg 2014;260(6):1128-1137.

41. Tatum PM, Jr., Harmon CM, Lorenz RG, Dimmitt RA. Toll-like receptor 4 is protective against neonatal murine ischemia-reperfusion intestinal injury. J Pediatr Surg 2010;45(6):1246-1255.

42. Richter JM, Schanbacher BL, Huang H, Xue J, Bauer JA, Giannone PJ. LPS-binding protein enables intestinal epithelial restitution despite LPS exposure. J Pediatr Gastroenterol Nutr 2012;54(5):639-644. 
43. Bates JM, Akerlund J, Mittge E, Guillemin K. Intestinal alkaline phosphatase detoxifies lipopolysaccharide and prevents inflammation in zebrafish in response to the gut microbiota. Cell Host Microbe 2007;2(6):371-382.

44. Rentea RM, Liedel JL, Welak SR et al. Intestinal alkaline phosphatase administration in newborns is protective of gut barrier function in a neonatal necrotizing enterocolitis rat model. $\mathrm{J}$ Pediatr Surg 2012;47(6):1135-1142.

45. Heinzerling NP, Liedel JL, Welak SR et al. Intestinal alkaline phosphatase is protective to the preterm rat pup intestine. J Pediatr Surg 2014;49(6):954-960.

46. Tremblay E, Ferretti E, Babakissa C et al. Gene-expression profile analysis in the mid-gestation human intestine discloses greater functional immaturity of the colon as compared with the ileum. J Pediatr Gastroenterol Nutr 2011;52(6):670-678.

47. Maheshwari A, Lacson A, Lu W et al. Interleukin-8/CXCL8 forms an autocrine loop in fetal intestinal mucosa. Pediatr Res 2004;56(2):240-249.

48. Nguyen DN, Sangild PT, Ostergaard MV, Bering SB, Chatterton DE. Transforming growth factor-beta2 and endotoxin interact to regulate homeostasis via interleukin-8 levels in the immature intestine. Am J Physiol Gastrointest Liver Physiol 2014;307(7):G689-G699.

49. Walker WA. Initial intestinal colonization in the human infant and immune homeostasis. Ann Nutr Metab 2013;63 Suppl 2:8-15. 
50. Cilieborg MS, Boye M, Sangild PT. Bacterial colonization and gut development in preterm neonates. Early Hum Dev 2012;88 Suppl 1:S41-S49.

51. Brower-Sinning R, Zhong D, Good M et al. Mucosa-associated bacterial diversity in necrotizing enterocolitis. PLoS ONE 2014;9(9):e105046. 


\section{Figure legends}

Figure 1. Expression of intestinal genes related to nutrient digestion and absorption (LCT, SGLT1), mucosal barrier function (MUC1, IAP), cytokine secretion (IL6, IL8) and LPS-mediated signaling $(L P B)$ in the small intestine of preterm and near-term pigs at birth, before any feeding ( $\mathrm{n}=17-18$, means $\pm \mathrm{SEM} ;{ }^{*} P<0.05,{ }^{* *} P<0.01,{ }^{* * *} P<0.001$ denote significant differences between groups).

Figure 2. Relative ex vivo D-glucose and L-leucine uptake in the proximal jejunum of newborn, unfed preterm and near-term pigs (A). Basal secretion of the pro-inflammatory cytokines IL-6, IL-8, and TNF- $\alpha$ in $24 \mathrm{~h}$ explants from the small intestine of newborn, unfed preterm and near-term pigs (B), and induced IL-8 secretion in explants incubated for $24 \mathrm{~h}$ with lipopolysaccharide (LPS, $10 \mathrm{ng} / \mathrm{ml}$ ),

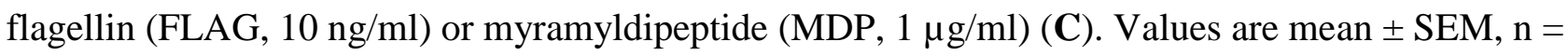
12-18. $* P<0.05,{ }^{* *} P<0.01$ : significant difference between groups.

Figure 3. Two day old formula- or colostrum-fed preterm and near-term pigs ( $\mathrm{n}=14-25$, means \pm SEM). Diet-dependent genes indicate genes that are differentially regulated only by diet (across gestational ages), whereas age-dependent genes indicate genes differentially regulated only by gestational age (across diets). Only genes that were significantly different between groups are shown $\left({ }^{*} P<0.05,{ }^{* *} P<0.01,{ }^{* * *} P<0.001\right.$ indicate significant differences between groups).

Figure 4. Small intestinal microbiology analyzed by 454 pyro sequencing. Mean relative abundance of the 15 most dominating OUTs in the distal small intestine according to treatment groups (A). Alpha diversity (analyses of Observed Species) estimating the mean number of OTUs based on 10-1000 reads per sample (B). Weighted PCoA plots of 454 pyro sequencing data showing three dimensional separation of pigs based on overall similarity (beta diversity) of the small intestinal microbiota 
according to (C) diet (white circles: colostrum; grey circles: formula) and (D) gestational age (white circles: preterm pigs; grey circles: near-term pigs).

Figure 5. In situ bacterial abundance based on FISH targeting eubacterial 16S rRNA. (A) Mean FISH scores for fed preterm and near-term pigs showing overall bacterial abundance in proximal, middle and distal small intestine. Tissue sections from the distal small intestine of (B) preterm colostrum-fed pigs, (C) preterm formula-fed pigs (with NEC), (D) near-term colostrum-fed pigs and (E) near-term formula-fed pigs. 
Figure 1

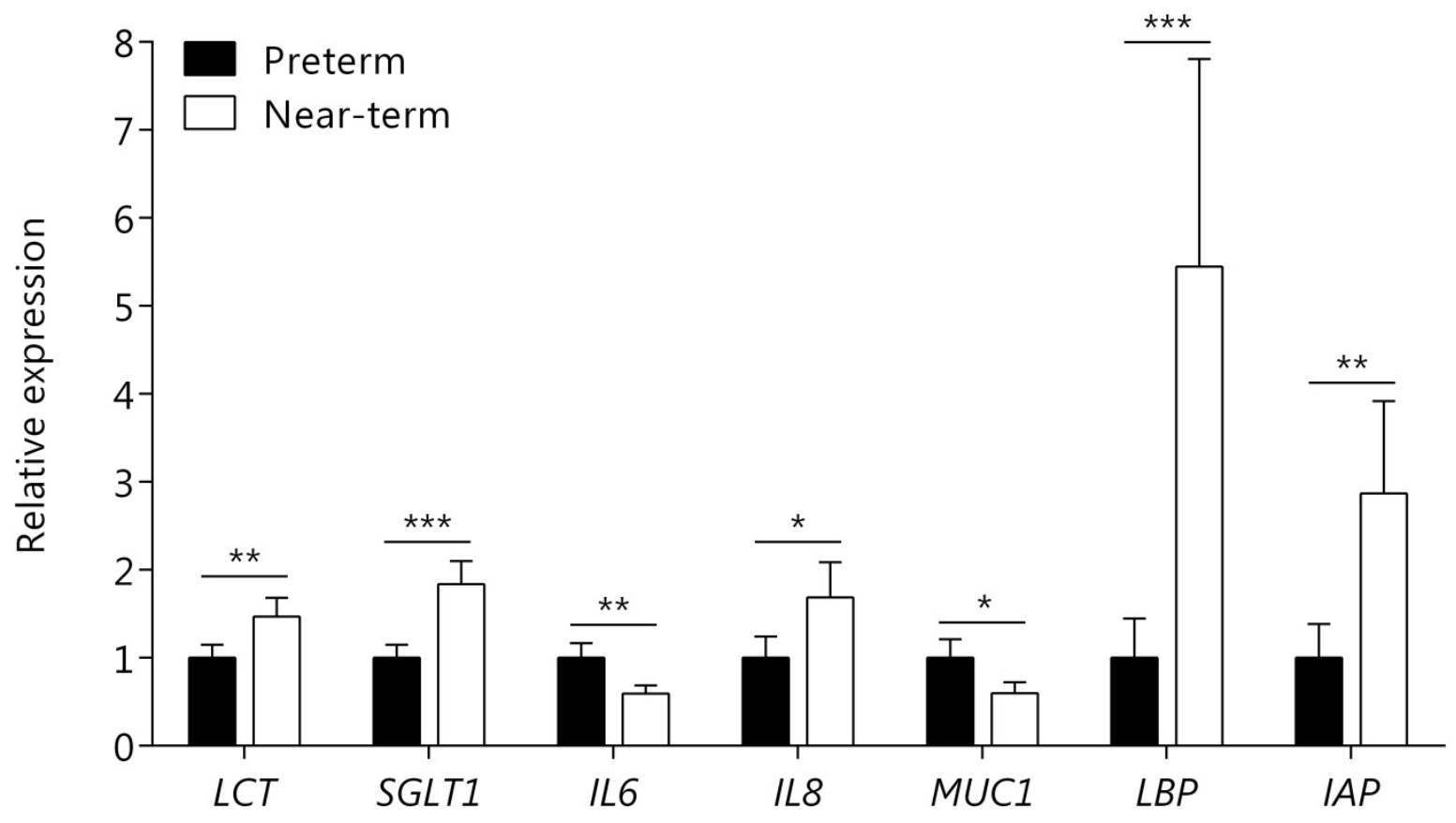


Figure 2
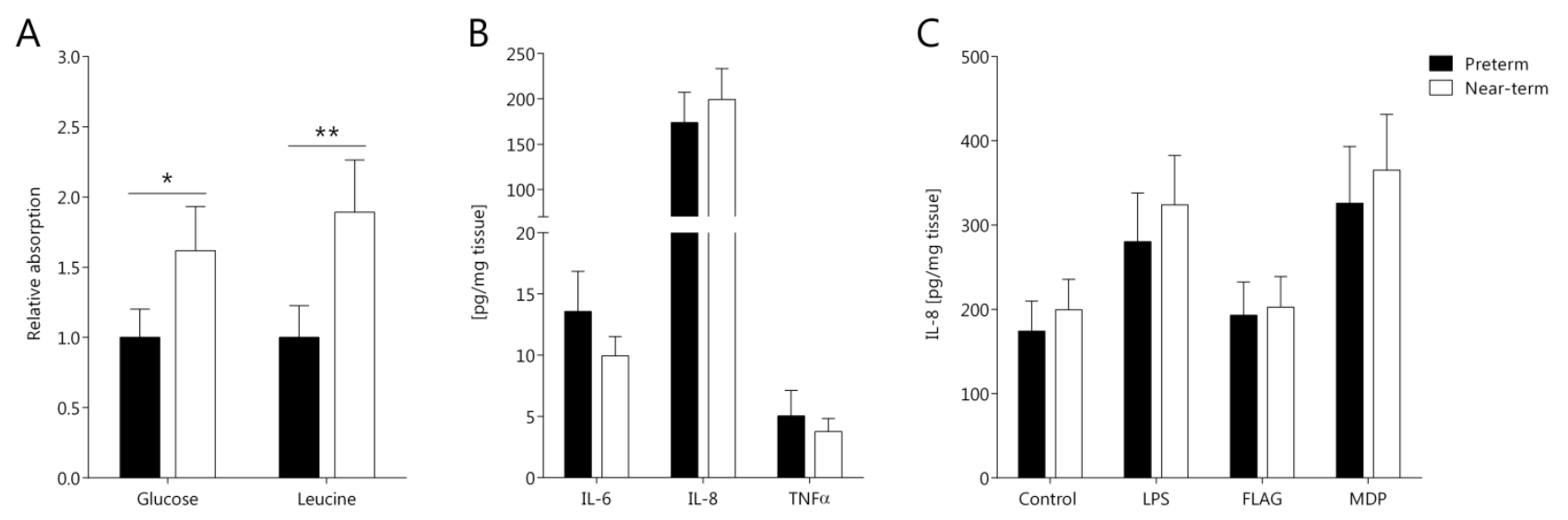
Figure 3

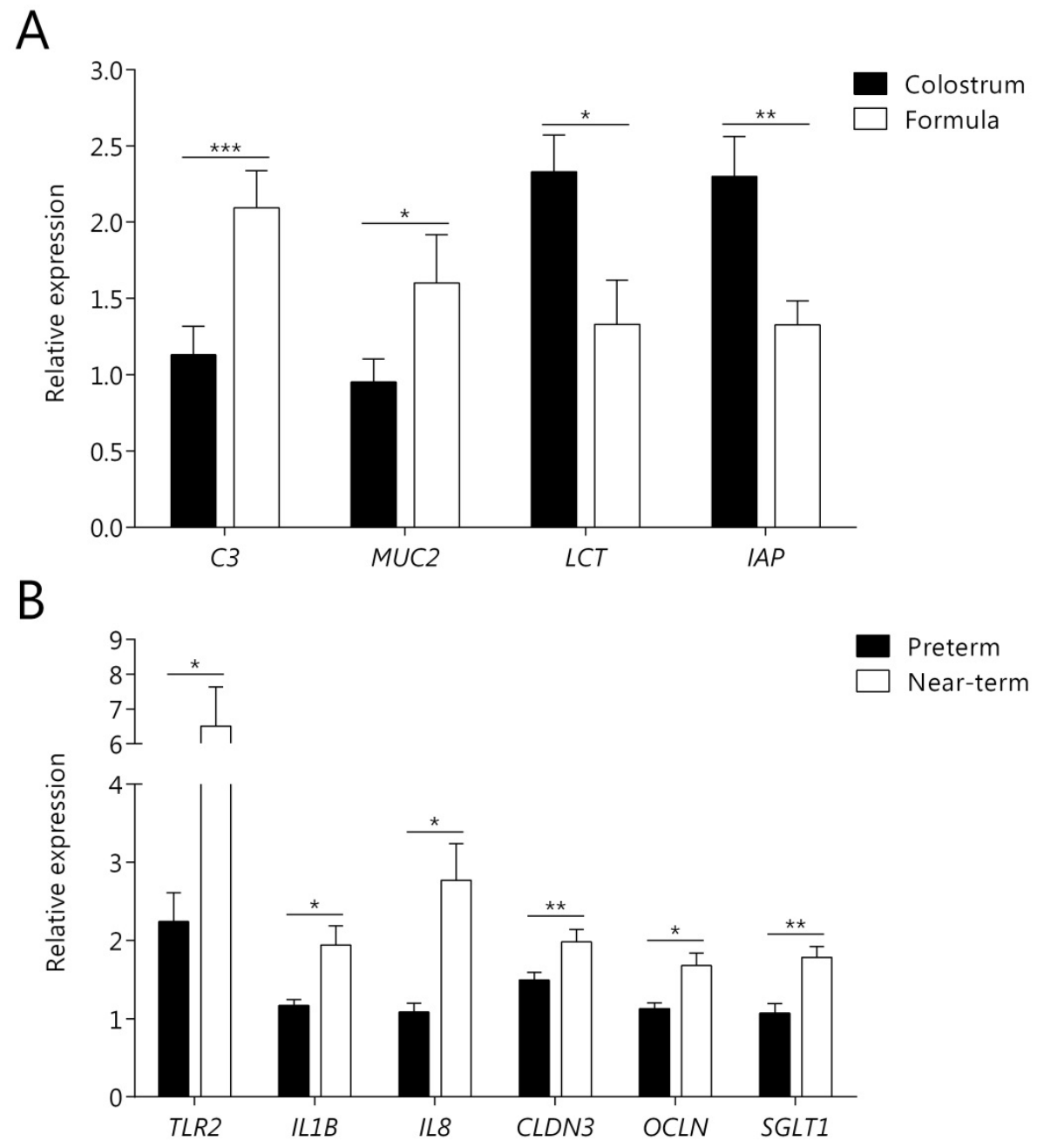


Figure 4
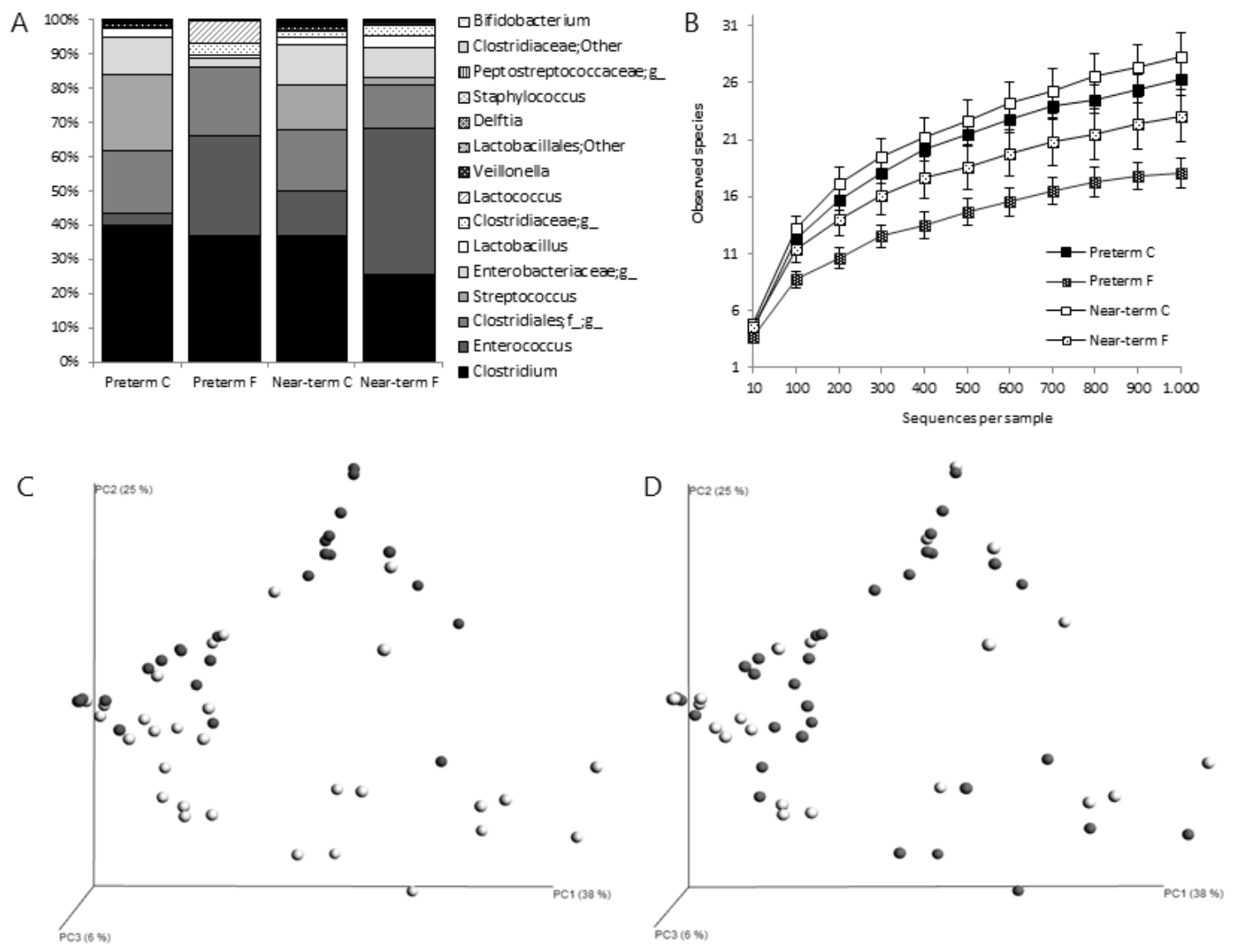
Figure 5

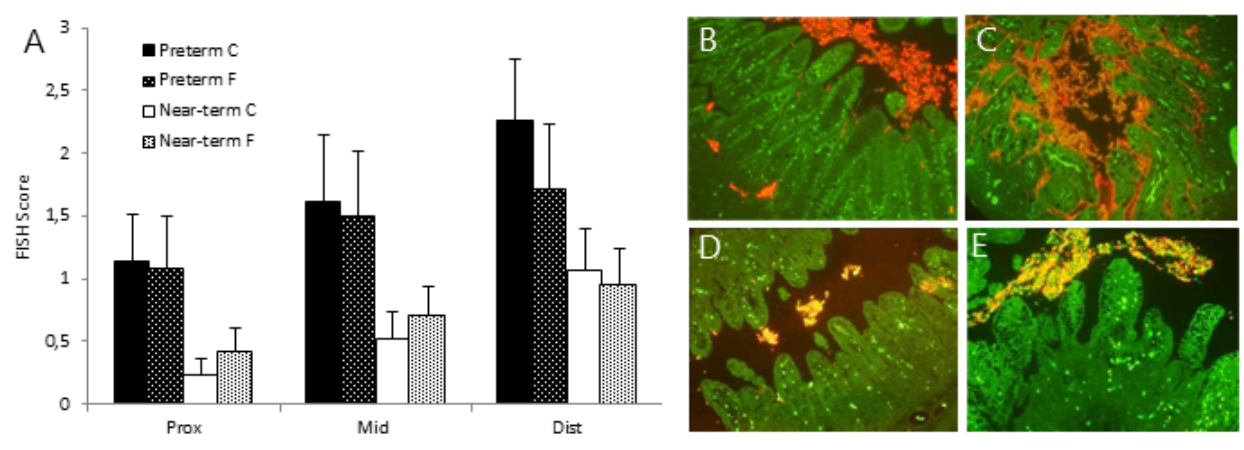

